\title{
Diabetes control is associated with environmental quality in the USA
}

\author{
Jyotsna S Jagai®1, Alison K Krajewski ${ }^{2,3}$, Kyla N Price ${ }^{4}$, Danelle T Lobdell ${ }^{3}$ and Robert M Sargis ${ }^{(1)}$ \\ 1Division of Environmental and Occupational Health Sciences, School of Public Health, University of Illinois at Chicago, Chicago, Illinois, USA \\ ${ }^{2}$ Oak Ridge Institute for Science and Education, U.S. Environmental Protection Agency, Center for Public Health and Environmental Assessment, Public \\ Health and Environmental Systems Division, Chapel Hill, North Carolina, USA \\ ${ }^{3}$ U.S. Environmental Protection Agency, Center for Public Health and Environmental Assessment, Research Triangle Park, North Carolina, USA \\ ${ }^{4}$ Division of Endocrinology, Diabetes, and Metabolism, Department of Medicine, University of Illinois at Chicago, Chicago, Illinois, USA
}

Correspondence should be addressed to J S Jagai: jjagai2@uic.edu

\begin{abstract}
Environmental parameters, including built and sociodemographic environments, can impact diabetes control (DC). Epidemiological studies have associated specific environmental factors with DC; however, the impact of multidimensional environmental status has not been assessed. The Environmental Quality Index (EQI), a comprehensive quantitative metric capturing five environmental domains, was considered as an exposure. Age-adjusted rates of DC prevalence for each county in the United States were used as an outcome. DC was defined as the proportion of adults aged $20+$ years with a previous diabetes diagnosis who currently do not have high fasting blood glucose ( $\geq 126 \mathrm{mg} / \mathrm{dL}$ ) or elevated HbA1c ( $\geq 6.5)$. We conducted county-level analyses of DC prevalence rates for the years 2004-2012 in association with EQI for 2006-2010 and domain-specific indices using random intercept multilevel linear regression models clustered by state and controlled for county-level rates of obesity and physical inactivity. Analyses were stratified by rural-urban strata, and results are reported as prevalence rate differences (PRD) with 95\% Cls comparing highest quintile/worst environmental quality to lowest quintile/best environmental quality. The association of DC with cumulative environmental quality was negative after control for all counties (PRD $-0.32,95 \% \mathrm{Cl}$ : $-0.38,-0.27)$; suggesting that rates of $D C$ worsen as environmental quality declines. While overall environmental quality exerts effects on DC that vary across the rural-urban spectrum, poor sociodemographic, and built environmental factors are associated with decreased DC nationally. These data suggest improvements in environmental quality mediated by larger-scale policy and practice interventions may improve glycemic control and reduce the morbidity and mortality arising from hyperglycemia.
\end{abstract}

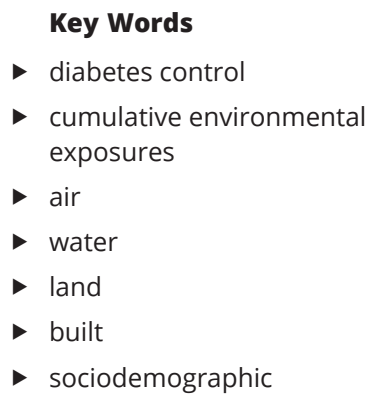

Endocrine Connections (2021) 10, 1018-1026

\section{Introduction}

Diabetes mellitus (DM) is a devastating metabolic disease that contributes significantly to individual and societal morbidity and mortality. In 2018, 34.2 million people, approximately $10.5 \%$ of the United States (US) population, were estimated to have diabetes, and it was the seventh leading cause of death (1). In 2017, the total cost of diagnosed diabetes alone in the US was estimated to be
$\$ 327$ billion (2). This tremendous impact of DM arises from its potent contribution to micro- and macrovascular disease, including retinopathy, nephropathy, and neuropathy as well as atherosclerotic cardiovascular disease (ASCVD) and heart failure. Importantly, clinical trials over the last 30 years have clearly demonstrated that improved glycemic control among those with both type 1

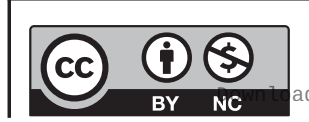

This work is licensed under a Creative Commons Attribution-NonCommercial 4.0 International License. ded from Bioscientifica.com at 04/26/2023 02:01:29PM 
and type 2 diabetes improves microvascular outcomes $(3,4)$. Moreover, recent clinical trials employing newer pharmacological approaches show improvements in macrovascular outcomes as well $(5,6)$. Thus, in addition to efforts to reduce the prevalence of diabetes, the toll of diabetes can be alleviated by improvements in glycemic control. However, despite advances in clinical care, a significant fraction of those with diabetes do not achieve glycemic control (7).

While clinical diabetes management focuses on lifestyle interventions and pharmacotherapy (8), emerging evidence linking environmental factors with diabetes pathogenesis raises important questions with regard to the capacity of environments to modulate diabetes risk and disease-associated outcomes. Over the last 2 decades, a variety of cell-based, animal, and epidemiological studies have linked various environmental toxicants with diabetes risk $(9,10)$. Furthermore, these exposures may partially explain the disproportionate burden of diabetes in ethnic and racial minorities as well as among those with low incomes (11). Evidence now also implicates multiple components of the built and social environments with diabetes risk (12). What is not well understood is how diverse environmental factors work in concert to mediate diabetes risk, and even less is known about how cumulative environmental factors influence diabetes control (DC). While clinical management of DM involves comprehensive management strategies directed at glycemic, blood pressure, and lipid targets among others (8), herein we focus on the specific influence of environments on glycemic control, namely the ability of those with diabetes to achieve glycemic targets known to reduce the risk of diabetes-associated complications.

To address these knowledge gaps, the goal of the present study was to understand the association between a comprehensive set of environmental factors and rates of DC. While some studies have identified potential environmental factors that contribute to DC, to the best of our knowledge, none provides a comprehensive assessment of various environmental domains. The Environmental Quality Index (EQI) was designed to provide a more comprehensive estimate of environmental quality in order to improve our understanding of how the environment affects health $(13,14)$. The EQI consists of data representative of five distinct environmental domains: air, water, land, built, and sociodemographic. Together, these variables are combined to form an overall composite score assigned to each individual county allowing for countylevel comparisons. Previous work has demonstrated that diabetes prevalence is strongly associated with sociodemographic environments nationally, while overall environmental quality, built environment, and land use demonstrated marked differences in associations across the urban-rural continuum which suggests varying drivers across rural-urban strata (15).

The present study expands upon this work to examine the potential role that environmental factors play in the marked geographic disparities in DC in the US as well as across the rural-urban spectrum. We examined countylevel rates of DC for the years 2004-2012 in association with the EQI for the years 2006-2010. We also considered associations with domain-specific indices to assess which domains, if any, drive associations with DC. Evidence that environments modulate DC may promote the development of environmental policy interventions to augment individual-level action to reduce the burden of diabetes.

\section{Methods}

\section{Study population}

Population-based county-level estimates for the prevalence of DC were obtained from the Institute for Health Metrics and Evaluation (IHME) for the years 2004-2012 (16). DC prevalence rate was defined as the proportion of people within a county who had previously been diagnosed with diabetes (fasting plasma glucose $\geq 126 \mathrm{mg} / \mathrm{dL}$, hemoglobin A1c $\left(\mathrm{HbA}_{1 \mathrm{c}}\right)$ of $\geq 6.5 \%$, or diabetes diagnosis) but do not currently have high fasting plasma glucose or $\mathrm{HbA}_{1 \mathrm{c}}$ for the period 2004-2012. DC estimates were directly derived from the modeled data as DC $=1$ - uncontrolled DM/diagnosed DM, where uncontrolled DM was defined as diagnosed DM and with high FPG ( $\geq 126 \mathrm{mg} / \mathrm{dL})$ and/or A1c $(\geq 6.5 \%)$ (Dwyer-Lindgren, personal communication). A complete description of the imputation methods used is provided in Dwyer-Lindgren et al. (16). Briefly, DC prevalence estimates were calculated using a two-stage approach. The first stage used National Health and Nutrition Examination Survey (NHANES) data to predict high fasting plasma glucose (FPG) levels ( $\geq 126 \mathrm{mg} / \mathrm{dL})$ and/or $\mathrm{HbA}_{1 \mathrm{C}}$ levels $(\geq 6.5 \%$ (48 mmol/mol)) based on self-reported demographic and behavioral characteristics (16). This model was then applied to Behavioral Risk Factor Surveillance System (BRFSS) data to impute high FPG and/or $\mathrm{HbA}_{1 \mathrm{C}}$ status for each BRFSS respondent (16). The second stage used the imputed BRFSS data to fit a series of small area models, which were used to predict the county-level prevalence of diabetes-related outcomes, including DC (16).

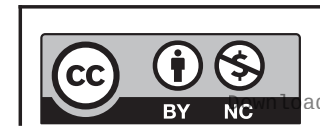

This work is licensed under a Creative Commons Attribution-NonCommercial 4.0 International License. ded from Bioscientifica.com at 04/26/2023 02:01:29PM 


\section{Exposure data: the Environmental Quality Index (EQI)}

The EQI was used as a metric of cumulative environmental exposures at the county level representing the period 2006-2010. We considered the 2006-2010 EQI to assess contemporaneous effects of environmental exposures. The EQI includes variables representing each of five environmental domains, air, water, land, built environment, and sociodemographic. The datasets included and the construction of the 2006-2010 EQI were similar to that of the 2000-2005. Datasets used and construction of the 2000-2005 are described elsewhere (13, 14). Briefly, for the 2006-2010, domain-specific indices (air index, water index, land index, built environment index, and sociodemographic index) were created by retaining the first component of a principal components analysis (PCA) that included all of the domain-specific variables. A list of variables and those used in the 2006-2010 EQI is provided (Supplementary Table 1, see section on supplementary materials given at the end of this article). The EQI was then created by retaining the first component of a PCA that combined the domain-specific indices. Recognizing environments differ across the rural-urban continuum, the EQI and domain-specific indices construction were stratified by rural-urban continuum codes (RUCC) (14). We utilized four categories for which RUCC1 represents metropolitan urbanized; RUCC2 non-metro urbanized; RUCC3 less urbanized; RUCC4 thinly populated, which have been used in previous health analyses (15). Finally, we have six non-stratified indices (one overall EQI and five domain-specific indices) and six corresponding indices for each of the four RUCC strata. This allows for the assessment of cumulative environmental exposure, domain-specific drivers, and rural-urban variations. For the domainspecific analysis, we valance corrected (i.e. corrected directionality) the domain-specific indices to ensure that the directionality of the variables was consistent with higher values suggesting poorer quality (more pollution).

\section{Covariates}

County-level data on obesity and leisure-time physical inactivity for the years 2004-2012, annually, were downloaded from the Centers for Disease Control and Prevention to use as covariates in analyses. These values are estimated from the Behavioral Risk Factor Surveillance System (BRFSS) data using Bayesian methods to statistically model estimates utilizing data from surrounding counties to strengthen estimates for individual counties.
Additionally, for domain-specific analyses, the other domains were included in the statistical models as covariates.

\section{Statistical analyses}

We used a random intercept mixed-effect linear model, clustered by state, to estimate the fixed effects of EQI quintiles and environmental domain-specific quintiles on DC prevalence annually. We clustered by the state in order to account for policies in funding for and care of diabetes which may vary at the state level. We conducted analyses using quintiles, which allow for more meaningful interpretation between areas of good (1st quintile), moderate (3rd quintile), and poor (5th quintile) environmental quality.

The regression equation for the random intercept mixed-effect linear model is:

$$
Y(i, j, k)=\beta_{0}+\beta_{1} X_{i, j}+b_{0 i}+b_{1 i} x_{i j}
$$

where $Y(i, j, k)$ is the outcome (diabetes control) for the $i$ th state, $j$ th county in the $k$ th year; $X_{i, j}$ is the exposure measurement (overall EQI or specific domain) for the $i$ th state and $j$ th county; $x_{i, j, k}$ are the covariates, county-level obesity and leisure-time physical inactivity, for the $i$ th state, $j$ th county, and the $k$ th year; $\beta_{0}$ is the overall intercept; $\beta_{1}$ is the overall slope; $b_{0 i, j}$ is the state and county intercept; $\mathrm{b}_{1, i j}$ is the state and county slope.

Results are reported as prevalence rate differences (PRD) with 95\% CIs comparing the highest quintile/ worst environmental quality to the lowest quintile/ best environmental quality exposure metrics. PRDs are representative of the entire period of interest, 2004-2012. Due to the availability of DC data and covariate data, not all counties were captured (nine counties missing data); however, the majority, 3134 of 3142 (99.7\%), were utilized in the analysis. DC prevalence data and covariate data for nine individual years (2004-2012) were used; therefore, the final regression model utilized data for 28,206 counties (3134 counties $\times 9$ years). All analyses were stratified by four rural-urban continuum codes to assess associations by rurality/urbanicity. Analyses were conducted using SAS (SAS Institute, Cary, NC; v9.4) statistical software, and figures were made using R (R Foundation for Statistical Computing, Vienna, Austria).

\section{Results}

Of the 3134 counties represented in the analysis, 34.7\% $(n=1088)$ were metropolitan urbanized (RUCC1), 
$10.3 \% \quad(n=323) \quad$ were non-metropolitan urbanized (RUCC2), 33.7\% ( $n=1056)$ were less urbanized (RUCC3), and $21.3 \%(n=667)$ were thinly populated (RUCC4). This mirrors the RUCC distribution of all US counties, which is 34\% RUCC1, 10\% RUCC2, 34\% RUCC3, and 21\% RUCC4. The average county-level prevalence rate of DC was 26.41 per 100,000 population (S.D. 1.29) for all counties.

The association of diabetes control, a positive outcome, with cumulative environmental quality is negative after controlling for obesity and leisure-time physical inactivity for all counties (PRD -0.32, 95\% CI: $-0.38,-0.27$ ) (Fig. 1), suggesting rates of DC decrease with worse environmental quality. Associations varied across environment-specific domains. The air domain was associated with an increased prevalence of DC (PRD 0.15, 95\% CI: 0.10, 0.20) (Fig. 1). The associations with the water and land domains were inverse. The strongest associations were seen with the sociodemographic domain (PRD -1.79, 95\% CI: -1.85, -1.73) (Fig. 1). The built domain also demonstrated inverse associations with the prevalence of DC. A summary of results for all counties by domain is shown in Table 1 .

Associations also varied across the rural-urban strata (summarized in Table 1). In the metropolitan urbanized counties (RUCC1), the association with cumulative environmental quality was strongly inverse (Fig. 2, panel A). Therefore, it suggests rates of DC decrease with worse environmental quality. The results for the air, water, and land domains were close to null in RUCC1. The built and sociodemographic domains demonstrated strong inverse associations with DC prevalence.

In the non-metropolitan urbanized counties (RUCC2), the association with cumulative environmental quality is strongly inverse (PRD $-1.20,95 \% \mathrm{CI}:-1.40,-1.00)$
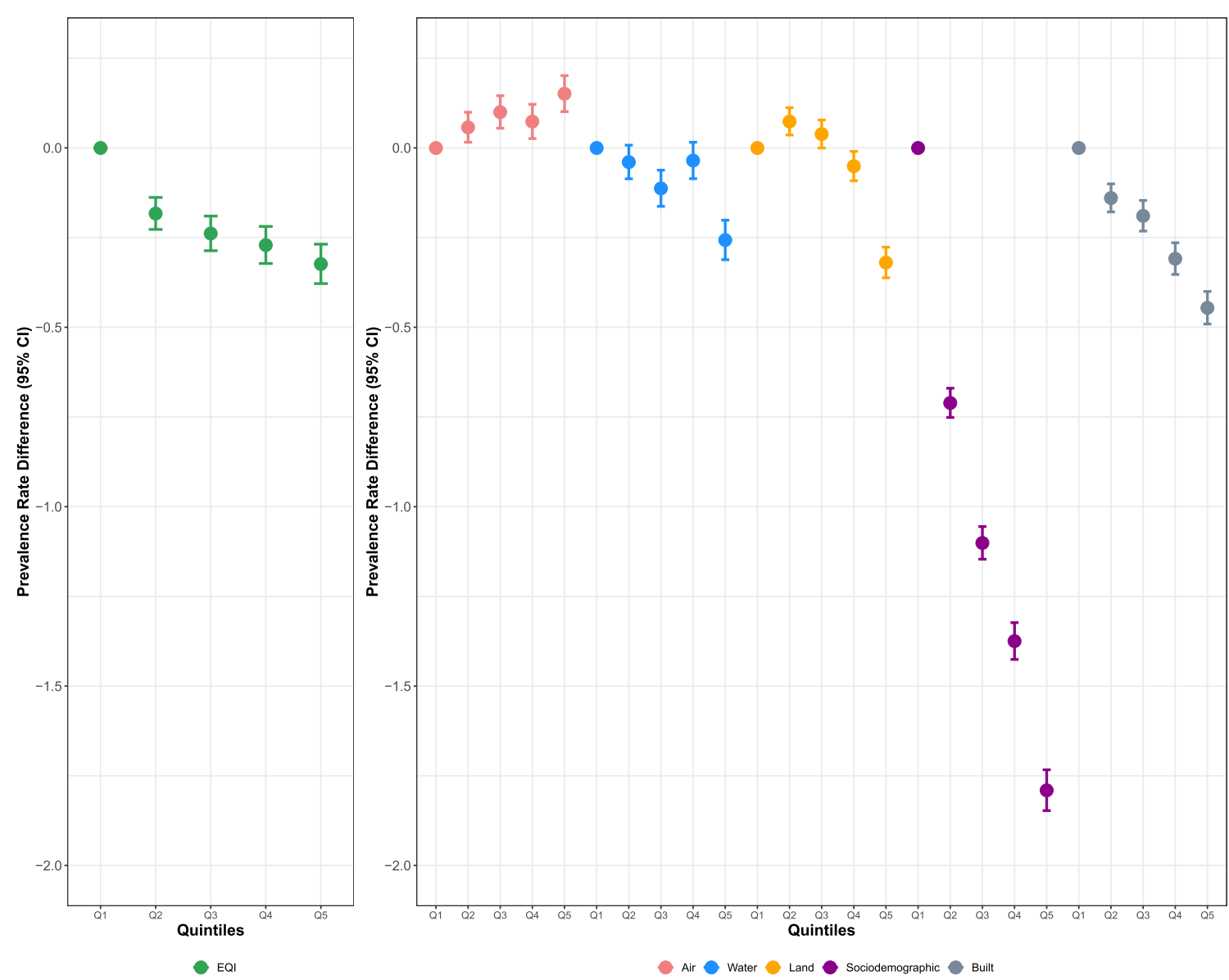

Figure 1

Diabetes control prevalence rate differences, in reference to quintile 1, with $95 \%$ Cls for all counties by quintiles of environmental quality index for the years 2006-2010 and domain-specific indices, controlling for obesity prevalence and leisure-time physical inactivity prevalence and all other domains for the domain-specific analyses.

https://ec.bioscientifica.com https://doi.org/10.1530/EC-21-0132 (c) 2021 The authors Published by Bioscientifica Ltd

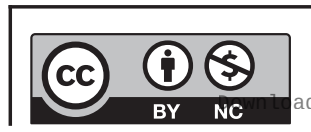

This work is licensed under a Creative Commons Attribution-NonCommercial 4.0 International License. ded from Bioscientifica.com at $04 / 26 / 2023$ 02:01:29PM via free access 
Table 1 Summary of results for overall EQI and all domains for 2006-2010 indices.

\begin{tabular}{|c|c|c|}
\hline \multirow[b]{2}{*}{ Poor } & \multicolumn{2}{|c|}{ 2006-2010 EQI } \\
\hline & $\begin{array}{l}\text { is associated } \\
\text { with__ rates for DC }\end{array}$ & for ... \\
\hline Overall environmental & $\downarrow$ & All counties \\
\hline quality (EQI) & $\downarrow$ & $\begin{array}{l}\text { RUCC1, RUCC2, } \\
\text { RUCC3, RUCC4 }\end{array}$ \\
\hline Air & $\frac{\downarrow}{\uparrow}$ & $\begin{array}{l}\text { All counties } \\
\text { RUCC1 } \\
\text { RUCC2, RUCC3, } \\
\text { RUCC4 }\end{array}$ \\
\hline Water & $\begin{array}{l}\downarrow \\
\downarrow\end{array}$ & $\begin{array}{l}\text { All counties } \\
\text { RUCC1, RUCC2, } \\
\text { RUCC3, RUCC4 }\end{array}$ \\
\hline Land & $\begin{array}{l}\downarrow \\
\downarrow \\
-\end{array}$ & $\begin{array}{l}\text { All counties } \\
\text { RUCC1 } \\
\text { RUCC2, RUCC3, } \\
\text { RUCC4 }\end{array}$ \\
\hline Sociodemographic & $\begin{array}{l}\downarrow \\
\downarrow\end{array}$ & $\begin{array}{l}\text { All counties } \\
\text { RUCC1, RUCC2, } \\
\text { RUCC3, RUCC4 }\end{array}$ \\
\hline Built & $\begin{array}{l}\downarrow \\
\downarrow\end{array}$ & $\begin{array}{l}\text { All counties } \\
\text { RUCC1, RUCC2, } \\
\text { RUCC3, RUCC4 }\end{array}$ \\
\hline
\end{tabular}

(Fig. 2, panel B). The air domain demonstrated associations with increased prevalence of DC. The water and land domains showed marginal associations with DC. The sociodemographic domain demonstrated an inverse association with DC.

In the less urbanized counties (RUCC3), cumulative environmental quality controlling for obesity and leisuretime physical inactivity was associated with decreased prevalence of DC (PRD -1.04, 95\% CI: -1.14, -0.94) (Fig. 2, panel C). When considering environmental domains, the air domain demonstrated associations with increased prevalence of DC. The water domain showed marginal associations with DC. The land domain demonstrated marginal inverse associations with DC. The built and sociodemographic domains demonstrated inverse associations with DC.

Lastly, in the thinly populated counties (RUCC4), cumulative environmental quality controlling for obesity and leisure-time physical inactivity was associated with decreased the prevalence of DC (PRD -0.92, 95\% CI: -1.06, -0.78) (Fig. 2, panel D). When considering environmental domains, the air, land, and water domains demonstrated marginal associations with prevalence of DC. The built and sociodemographic domains showed inverse associations with the EQI. Results for all quintiles, all domains, and all RUCC with coefficients and 95\% CIs for all variables in the model are shown in Supplementary Table 2.

\section{Discussion}

Existing evidence suggests that built and sociodemographic environments can impact the control of diabetes, but the effect of cumulative environmental exposures has not been considered. To address this gap in research, we utilized a comprehensive measure of environmental quality derived from publicly available data sets that quantify environmental exposures for the period 2006-2010. We found that decreases in cumulative environmental quality were associated with decreases in the prevalence of DC. Using the 2006-2010 EQI, we found that the prevalence of DC decreased as environmental quality decreased with a prevalence rate difference of -0.32 ; suggesting that if a county were to improve from the quintile with the poorest environmental quality to that with the best environmental quality, the prevalence of diabetes control will improve by 0.32 per 100,000 population. This association remained for all rural/urban strata, demonstrating that poor environmental exposures were associated with reduced rates of DC. These data suggest that environmental quality influences DC, and policies that improve environmental quality may improve glycemic control and lower the morbidity associated with diabetes.

Associations varied by environmental domain as well as with the strongest associations seen in the sociodemographic domain for all rural/urban strata. Within the sociodemographic domain of the EQI, there are 12 different variables derived from 4 data sources, the United States Census, Federal Bureau of Investigation (FBI) Uniform Crime Report (UCR), Leip's Atlas of the United Sstates Presidential Elections, and United States Department of Agriculture (17, 18, 19, 20). These variables include the percentages of renter occupied spaces, vacant units, percent of vacant housing units, median household value, median household income, persons living under the poverty level, unemployed persons, number of occupants per room, percentage of individuals with Bachelor's degree or higher as well as income inequality, percent of the county voting Democrat, percent of county employed in a creative class, and rates of violent crime. In comparing the factors within the sociodemographic domain to rates of diabetes control, there is a decrease in DC with worsening sociodemographic domain qualities overall and for RUCC1, RUCC3, and RUCC4 specifically. These findings across the nation align with previously published studies examining specific sociodemographic components and composite metrics.

In attempting to dissect the sociodemographic domain variables, one factor that has been relatively well studied is

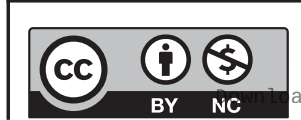



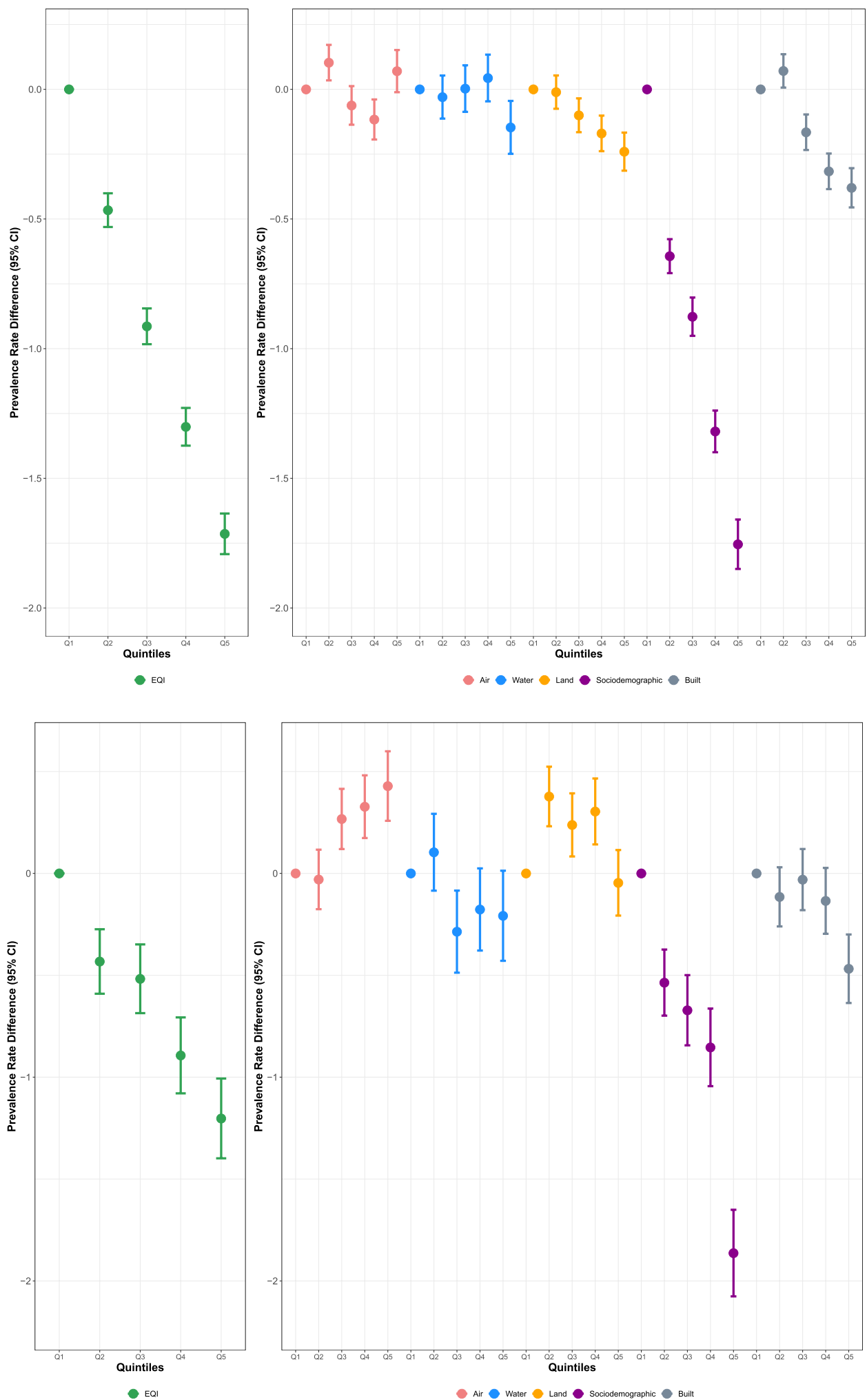

Figure 2

Diabetes control prevalence rate differences, in reference to quintile 1, with $95 \%$ Cls for metropolitan urbanized counties (RUCC1) (panel A), nonmetropolitan urbanized counties (RUCC2) (panel B), less urbanized counties (RUCC3) (panel C), thinly populated counties (RUCC4) (panel D) by quintiles of environmental quality index for 2006-2010 and domain-specific indices, controlling for obesity prevalence and leisure-time physical inactivity prevalence and all other domains for the domain-specific analyses.

https://ec.bioscientifica.com

https://doi.org/10.1530/EC-21-0132 (c) 2021 The authors Published by Bioscientifica Ltd

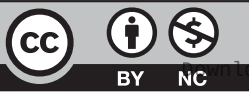

This work is licensed under a Creative Commons Attribution-NonCommercial 4.0 International License.

ded from Bioscientifica,com at 04/26/2023 02:01:29PM 
the relationship between food security and DC $(21,22,23$, 24). The United States Department of Agriculture (USDA) defines food insecurity as a lack of consistent access to enough food for an active, healthy lifestyle (21). Food insecurity is associated with diabetes rates as well as with poorer glycemic control (22). Behaviors associated with food insecurity include changes in eating habits, such as replacing healthier, more expensive foods with lower-cost, high-calorie options. Many food-insecure households also go through cycles of food scarcity and adequate food supplies leading to overconsumption during times of adequacy and underconsumption during times of scarcity (23). These cyclical patterns place patients at increased risk for adverse clinical outcomes including episodes of hyperglycemia and hypoglycemia and complications that negatively impact DC. In contrast, participants in the Supplemental Nutrition Assistance Program (SNAP), a federal program that is administered at the state level, for low-income families that provide funds that can be used toward healthier food purchases, had better DC compared to food-insecure counterparts that did not participate in SNAP (24). In addition to effects on food consumption, food-insecure individuals are also significantly more likely to scrimp on medications (25). Medication scrimping behaviors include delaying filling prescriptions, inability to afford medications, skipping medication doses, or taking less medication than recommended.

Another potential sociodemographic contributor to worse glycemic control is educational attainment and consequential health literacy. In one study exploring the impact of health literacy for patients with hypertension and diabetes in two urban public hospitals, patients with greater health literacy were more likely to recognize symptoms of hypoglycemia, know how to treat it, and possess greater disease knowledge; there was no relationship between literacy and the number of diabetes classes attended (26). There was a trend toward worse glucose control among those with lower literacy; however, the relationship did not reach statistical significance (26). In addition, education discrimination more broadly has been associated with poorer glycemic control and potentially worse health outcomes (27). Indeed, patients who reported perceived education discrimination had $\mathrm{HbA}_{1 \mathrm{c}}$ levels that were $0.5 \%$ higher.

In addition to relationships observed in the sociodemographic domain, lower rates of DC were also associated with worse land and built environments. The associations with the land were unexpected as there is little previous research into the impacts of exposures considered in the land domain and DC. However, there is evidence that neighborhood factors, the built environment, are associated with DC. Two studies have explored the relationship between composite neighborhood characteristics and cardiometabolic risk factors. The first study employed the Neighborhood Deprivation Index (NDI), which is composed of eight census-derived variables, including some of the same variables used to develop the EQI (12). In an analysis of 19 northern California counties, after adjusting for individual factors including income and education, the NDI was associated with poorer glycemic control as assessed by $\mathrm{HbA}_{1 \mathrm{c}}$ (12). In a similar study conducted among older women, there was a trend toward improved $\mathrm{HbA}_{1 \mathrm{c}}$ as neighborhood quality improved, although the results did not reach statistical significance (28).

The air domain and the water domain demonstrated marginal associations with the prevalence of DC. The variables included in the water domain have not been previously associated with DC. However, we were expecting a stronger association between the air domain and DC. Previous studies have suggested that current exposure to air pollution may affect one's ability to exercise outside or to access healthy foods, thereby affecting blood sugars $(29,30)$. This marginal effect between the air domain and DC is likely due to the use of county-level data. The county is a large heterogenous area, and the localized impacts of poor air quality are likely to be diluted over the spatial area of a county.

The EQI is a metric of cumulative environmental exposures that was developed utilizing publicly available data. However, environmental data are typically collected for administrative and regulatory purposes and, therefore, may not provide the spatial and/or temporal coverage to properly assess health outcomes (31). Many variables in the built and sociodemographic domain are associated with DC, and while we did see strong associations with the sociodemographic domain, the associations with the built environment were not as strong. This may be due to the resolution of the data in the built domain. Additionally, environmental data better represent urban areas compared to suburban and rural areas. Several of the factors included in the exposure metric as well as the outcome of countylevel rates of DC demonstrate spatial relationships. We did not account for any spatial associations in our analyses. These factors may demonstrate clustering effects that should be considered and accounted for in future analyses. Moreover, such analyses will be further augmented by finer spatial resolution for both exposure and outcome metrics.

The application of broad ecological exposure metrics like the EQI provides new insights into the impact 
of cumulative environmental exposures on DC. The EQI considers hundreds of environmental exposures simultaneously across multiple environmental domains, including the sociodemographic environment, which is often neglected when considering environmental exposures. In addition, we were able to leverage publicly available exposure and outcome data to assess relationships between environmental quality and DC on a national level. These data provide intriguing insights that should prompt targeted investigations examining how socioeconomic and other environmental drivers can affect glycemic control among those with diabetes and how these factors vary across the urban-rural continuum to better tailor place-based intervention strategies to specific communities in order to lessen the devastating toll of diabetes on individuals and society at large.

\section{Supplementary materials}

This is linked to the online version of the paper at https://doi.org/10.1530/ EC-21-0132.

\section{Declaration of interest}

The authors declare that there is no conflict of interest that could be perceived as prejudicing the impartiality of the research reported.

\section{Funding}

This work was supported by the National Institutes of Health (P30 DK092949 to J S J (via the Chicago Center for Diabetes Translational Research), P30 ES027792 (ChicAgo Center on Health and EnvironmenT), and R01 ES028879 (to R M S)), the American Diabetes Association (1-17JDF-033 to R M S), and the United States Environmental Protection Agency (EP-17-D-000063 to J S J). A K K supported in part by an appointment to the Internship/Research Participation Program at Office of Research and Development (Center for Public Health \& Environmental Assessment), the United States Environmental Protection Agency, administered by the Oak Ridge Institute for Science and Education through an interagency agreement between the United States Department of Energy and the United States Environmental Protection Agency.

\section{Disclaimer}

The views expressed in this manuscript are those of the authors and do not necessarily reflect the views or policies of the United States Environmental Protection Agency. Mention of trade names or commercial products does not constitute endorsement or recommendation for use.

\section{Author contribution statement}

J S J conceived the study and led the writing of the manuscript. A K K led the analysis and contributed to the writing of the manuscript. K N P assisted in the literature review and manuscript preparation. D T L led the team that developed the county-level estimates of environmental quality. R M S assisted in the conception and development of the study design, interpretation of results, and contributed to the writing of the manuscript.

\section{References}

1 National Diabetes Statistics Report 2020. Estimates of Diabetes and Its Burden in the United States 202032

2 American Diabetes Association. Economic costs of diabetes in the U.S. in 2017. Diabetes Care 201841 917-928. (https://doi.org/10.2337/dci180007)

3 King P, Peacock I \& Donnelly R. The UK Prospective Diabetes Study (UKPDS): clinical and therapeutic implications for type 2 diabetes. British Journal of Clinical Pharmacology 199948 643-648. (https://doi. org/10.1046/j.1365-2125.1999.00092.x)

4 Nathan DM \& DCCT/EDIC Research Group. The diabetes control and complications trial/epidemiology of diabetes interventions and complications study at 30 years: overview. Diabetes Care 2014 37 9-16. (https://doi.org/10.2337/dc13-2112)

5 Marso SP, Daniels GH, Brown-Frandsen K, Kristensen P, Mann JFE, Nauck MA, Nissen SE, Pocock S, Poulter NR, Ravn LS, et al. Liraglutide and cardiovascular outcomes in Type 2 diabetes. New England Journal of Medicine 2016375 311-322. (https://doi.org/10.1056/NEJMoa1603827)

6 Zinman B, Wanner C, Lachin JM, Fitchett D, Bluhmki E, Hantel S, Mattheus M, Devins T, Johansen OE, Woerle HJ, et al. Empagliflozin, cardiovascular outcomes, and mortality in type 2 diabetes. New England Journal of Medicine 2015373 2117-2128. (https://doi. org/10.1056/NEJMoa1504720)

7 Shaya FT, Yan X, Lin PJ, Simoni-Wastila L, Bron M, Baran R \& Donner TW. US trends in glycemic control, treatment, and comorbidity burden in patients with diabetes. Journal of Clinical Hypertension 201012 826-832. (https://doi.org/10.1111/j.17517176.2010.00365.x)

8 American Association of Clinical Endocrinologists. Comprehensive type 2 diabetes management algorithm. (available at: https://www. aace.com/disease-state-resources/diabetes/clinical-practice-guidelinestreatment-algorithms/comprehensive)

9 Heindel JJ, Blumberg B, Cave M, Machtinger R, Mantovani A, Mendez MA, Nadal A, Palanza P, Panzica G, Sargis RM, et al. Metabolism disrupting chemicals and metabolic disorders. Reproductive Toxicology 201768 3-33. (https://doi.org/10.1016/j. reprotox.2016.10.001)

10 Neel BA \& Sargis RM. The paradox of progress: environmental disruption of metabolism and the diabetes epidemic. Diabetes 201160 1838-1848. (https://doi.org/10.2337/db11-0153)

11 Ruiz D, Becerra M, Jagai JS, Ard K \& Sargis RM. Disparities in environmental exposures to endocrine-disrupting chemicals and diabetes risk in vulnerable populations. Diabetes Care 201841 193-205. (https://doi.org/10.2337/dc16-2765)

12 Laraia BA, Karter AJ, Warton EM, Schillinger D, Moffet HH \& Adler N. Place matters: neighborhood deprivation and cardiometabolic risk factors in the diabetes study of Northern California (DISTANCE). Social Science and Medicine 201274 1082-1090. (https://doi. org/10.1016/j.socscimed.2011.11.036)

13 Lobdell DT, Jagai JS, Rappazzo K \& Messer LC. Data sources for an environmental quality index: availability, quality, and utility. American Journal of Public Health 2011101 (Supplement 1) S277-S285. (https://doi.org/10.2105/AJPH.2011.300184)

14 Messer LC, Jagai JS, Rappazzo KM \& Lobdell DT. Construction of an environmental quality index for public health research. Environmental Health: A Global Access Science Source 201413 39. (https://doi. org/10.1186/1476-069X-13-39)

15 Jagai JS, Krajewski AK, Shaikh S, Lobdell DT \& Sargis RM. Association between environmental quality and diabetes in the USA. Journal of Diabetes Investigation 202011 315-324. (https://doi.org/10.1111/ jdi.13152)

16 Dwyer-Lindgren L, Mackenbach JP, Lenthe FJ van, Flaxman AD \& Mokdad AH. Diagnosed and undiagnosed diabetes prevalence by county in the U.S., 1999-2012. Diabetes Care 201639 1556-1562. (https://doi.org/10.2337/dc16-0678)

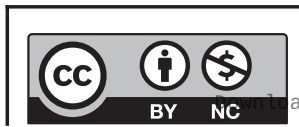

This work is licensed under a Creative Commons Attribution-NonCommercial 4.0 International License. ded from Bioscientifica.com at 04/26/2023 02:01:29PM 
17 Explore Census Data. (available at: https://data.census.gov/cedsci/)

18 Crime Data Explorer. (available at: https://crime-data-explorer. fr.cloud.gov/)

19 Dave Leip's Atlas of U.S. Presidential Elections. (available at: https:// uselectionatlas.org/)

20 USDA ERS - Creative Class County Codes. (available at: https://www. ers.usda.gov/data-products/creative-class-county-codes/)

21 Coleman-Jensen A, Rabbitt MP, Gregory CA \& Singh A. Household Food Security in the United States in 2018. Economic Research Report No. (ERR-270), 47 pp, 2018.

22 Berkowitz SA, Baggett TP, Wexler DJ, Huskey KW \& Wee CC. Food insecurity and metabolic control among U.S. adults with diabetes. Diabetes Care 201336 3093-3099. (https://doi.org/10.2337/dc130570)

23 Seligman HK \& Schillinger D. Hunger and socioeconomic disparities in chronic disease. New England Journal of Medicine 2010363 6-9. (https://doi.org/10.1056/NEJMp1000072)

24 Mayer VL, McDonough K, Seligman H, Mitra N \& Long JA. Food insecurity, coping strategies and glucose control in low-income patients with diabetes. Public Health Nutrition 201619 1103-1111. (https://doi.org/10.1017/S1368980015002323)

25 Knight CK, Probst JC, Liese AD, Sercy E \& Jones SJ. Household food insecurity and medication "scrimping" among US adults with diabetes. Preventive Medicine 201683 41-45. (https://doi.org/10.1016/j. ypmed.2015.11.031)
26 Williams MV, Baker DW, Parker RM \& Nurss JR. Relationship of functional health literacy to patients' knowledge of their chronic disease: a study of patients with hypertension and diabetes. Archives of Internal Medicine 1998158 166-172. (https://doi.org/10.1001/archinte.158.2.166)

27 Brice Reynolds DB, Walker RJ, Campbell JA \& Egede LE. Differential effect of race, education, gender, and language discrimination on glycemic control in adults with Type 2 diabetes. Diabetes Technology and Therapeutics 201517 243-247. (https://doi.org/10.1089/dia.2014.0285)

28 Corriere MD, Yao W, Xue QL, Cappola AR, Fried LP, Thorpe RJ, Szanton SL \& Kalyani RR. The association of neighborhood characteristics with obesity and metabolic conditions in older women. Journal of Nutrition, Health and Aging 201418 792-798. (https://doi. org/10.1007/s12603-014-0477-5)

29 Thiering E \& Heinrich J. Epidemiology of air pollution and diabetes. Trends in Endocrinology and Metabolism 201526 384-394. (https://doi. org/10.1016/j.tem.2015.05.002)

30 Yang BY, Fan S, Thiering E, Seissler J, Nowak D, Dong GH \& Heinrich J. Ambient air pollution and diabetes: a systematic review and metaanalysis. Environmental Research 2020180 108817. (https://doi. org/10.1016/j.envres.2019.108817)

31 Jagai JS, Rosenbaum BJ, Pierson SM, Messer LC, Rappazzo K, Naumova EN \& Lobdell DT. Putting regulatory data to work at the service of public health: utilizing data collected under the clean water act. Water Quality, Exposure and Health 20135 117-125. (https://doi. org/10.1007/s12403-013-0095-1)

Received in final form 27 July 2021

Accepted 3 August 2021

Accepted Manuscript published online 3 August 2021 https://ec.bioscientifica.com

https://doi.org/10.1530/EC-21-0132 (c) 2021 The authors Published by Bioscientifica Ltd
This work is licensed under a Creative Commons Attribution-NonCommercial 4.0 International License. ded from Bioscientifica.com at 04/26/2023 02:01:29PM 